\title{
Protective Role of Endogenous Carbon Monoxide in Hepatic Microcirculatory Dysfunction after Hemorrhagic Shock in Rats
}

\author{
Benedikt H.J. Pannen, ${ }^{\star}$ Nicola Köhler, ${ }^{\star}$ Burkhard Hole, ${ }^{\star}$ Michael Bauer, ${ }^{\ddagger}$ Mark G. Clemens, ${ }^{\S}$ and Klaus K. Geiger \\ $*$ Department of Anesthesiology and Critical Care Medicine, University of Freiburg, D-79106 Freiburg, Germany; ${ }^{\ddagger}$ Department of \\ Anesthesiology and Critical Care Medicine, University of the Saarland, D-66421 Homburg, Germany; and ${ }^{\S}$ Department of Biology, The \\ University of North Carolina at Charlotte, Charlotte, North Carolina 28223
}

\begin{abstract}
Maintenance of hepatic microcirculatory flow after ischemia of the liver is essential to prevent hepatic dysfunction. Thus, we determined the differential role of carbon monoxide (CO) and nitric oxide (NO) in the intrinsic control of sinusoidal perfusion, mitochondrial redox state, and bile production in the isolated perfused rat liver after hemorrhagic shock. Administration of tin protoporphyrin-IX $(50 \mu \mathrm{M})$, a specific inhibitor of the CO generating enzyme heme oxygenase, caused a decrease in sinusoidal flow that was more pronounced after shock compared with sham shock, as determined by in situ epifluorescence microscopy. This was associated with a shift in hepatocellular redox potential to a more reduced state (increased fluorescence intensity of reduced pyridine nucleotides in hepatocytes, decreased acetoacetate/ $\beta$-hydroxybutyrate ratio in the perfusate) and a profound reduction in bile flow. In sharp contrast, the preferential inhibitor of the inducible isoform of NO synthase S-methylisothiourea sulfate $(100 \mu \mathrm{M})$ did not affect sinusoidal flow, hepatic redox state, or function. This indicates that 1.) endogenously generated $\mathrm{CO}$ preserves sinusoidal perfusion after hemorrhagic shock, 2.) protection of the hepatic microcirculation by $\mathrm{CO}$ may serve to limit shock-induced liver dysfunction, and 3.) in contrast to $\mathrm{CO}$, inducible NO synthase-derived NO is of only minor importance for the intrinsic control of hepatic perfusion and function under these conditions. (J. Clin Invest. 1998. 102:12201228.) Key words: liver - microcirculation - nitric oxide • heme oxygenase $\bullet$ oxidation reduction
\end{abstract}

\section{Introduction}

Accumulating evidence suggests that maintenance of hepatic microcirculatory flow after ischemia of the liver, e.g., during resuscitation from hemorrhagic shock (HS), ${ }^{1}$ is essential to prevent the subsequent development of hepatic dysfunction or failure. For example, sinusoidal perfusion failure has been

Address correspondence to Benedikt H.J. Pannen, M.D., Anaesthesiologische Universitaetsklinik, Hugstetter Strasse 55, D-79106 Freiburg, Germany. Phone: +49-761-270-2306; FAX: +49-761-270-2396; E-mail: pannen@nz11.ukl.uni-freiburg.de

Received for publication 13 March 1998 and accepted in revised form 22 June 1998.

J. Clin. Invest.

(C) The American Society for Clinical Investigation, Inc. 0021-9738/98/09/1220/09 \$2.00

Volume 102, Number 6, September 1998, 1220-1228

http://www.jci.org shown to correlate with enzyme release from the liver and hepatocyte necrosis after HS (1). Moreover, we have previously demonstrated that physical prevention of microvascular shutdown using a flow-controlled reperfusion mode largely prevented parenchymal cell necrosis after ischemia/reperfusion of the liver, i.e., the degree of microcirculatory failure determined the extent of lethal hepatocyte injury (2). Based on the central role the liver plays in the whole organism's metabolic and immunologic response to injury (3), identification of the vasodilatory mediators that act to maintain sinusoidal flow after HS is of particular interest.

Nitric oxide (NO), a gaseous mediator that accounts for the biological activity of endothelium-derived relaxing factor (4), has been shown to play an indispensable role in reducing vascular resistance, thus keeping organ blood flow in a physiologic range. Such an NO-mediated vasodilatory action, which involves activation of soluble guanylate cyclase, has been demonstrated in multiple vascular beds (5), including the hepatic circulation (6). While there is only a small (7) or no NO-mediated vasodilatory effect in the hepatic microcirculation under physiologic conditions (8), recent studies have shown that increased generation of NO by either the constitutive (7) or the inducible isoform of NO synthase (iNOS; 8, 9), serves a protective role in minimizing hepatic microcirculatory dysfunction after endotoxemic shock. However, while Thiemermann et al. (10) have implicated increased iNOS activity in the vascular hyporeactivity and hemodynamic decompensation frequently associated with HS, Wang and colleagues (11) reported that the release of endothelium-derived relaxing factor from aortic rings is reduced after resuscitation from HS. In addition, decreases in plasma levels of the stable end products of NO metabolism have been observed after hemorrhage and resuscitation in humans (12). Therefore, the functional significance of iNOS-derived NO for the regulation of systemic vascular tone after HS remains controversial, and its role in the control of hepatic sinusoidal perfusion under these conditions is unknown.

More recently, much attention has been paid to the biological actions of carbon monoxide ( $\mathrm{CO}$ ), another gaseous molecule, generated in the body during physiological degradation of heme (iron protoporphyrin-IX) to biliverdin by heme oxy-

1. Abbreviations used in this paper: AcAc, acetoacetate; $\beta \mathrm{OHB}$, $\beta$-hydroxybutyrate; $\mathrm{CO}$, carbon monoxide; $\mathrm{D}_{\mathrm{s}}$, sinusoid diameter; $\mathrm{HO}$, heme oxygenase; HS, hemorrhagic shock; iNOS, inducible nitric oxide synthase; KHB, Krebs-Henseleit bicarbonate buffer; $\mathrm{NAD}(\mathrm{P}) \mathrm{H}$, reduced pyridine nucleotides; $\mathrm{NO}$, nitric oxide; $\mathrm{P}_{\mathrm{i}}$, inlet pressure; $\mathrm{P}_{\mathrm{o}}$, outlet pressure; $Q_{t}$, total portal resistance; $Q_{v}$, sinusoidal volumetric flow; $\mathrm{R}_{\mathrm{s}}$, sinusoid resistance; $\mathrm{R}_{\mathrm{t}}$, total portal resistance; SMT, S-methylisothiourea sulfate; SnPP-IX, tin protoporphyrin-IX; $\mathrm{V}_{\mathrm{RBC}}$, red blood cell velocity in sinusoids. 
genase (HO; EC 1.14.99.3) (13). Like NO, CO serves as an activator of soluble guanylate cyclase $(14,15)$. Since the liver is one of the most abundant sources of $\mathrm{HO}$ activity among organs (13), it is interesting to note that Suematsu et al. reported that blockade of $\mathrm{HO}$ in perfused livers isolated from normal rats increased not only total portal vascular resistance $\left(R_{t}\right)(16$, $17)$ but also caused constriction of hepatic sinusoids $(17,18)$. However, controversy exists whether $\mathrm{CO}$ may be of functional importance for the control of hepatic vascular resistance under pathophysiologic conditions $(18,19)$. In support of this hypothesis, we have recently demonstrated that HS results in enhanced hepatic expression of $\mathrm{HO}-1$, the stress-inducible isoform of the CO-generating enzymes $(20,21)$. Furthermore, the increase in $\mathrm{R}_{\mathrm{t}}$ upon blockade of the HO-CO pathway was much more pronounced after HS compared with sham controls (20). However, the effects of $\mathrm{CO}$ on sinusoidal perfusion after HS and the microvascular mechanisms through which $\mathrm{CO}$ may modulate resistance under these conditions have not yet been studied.

Thus, it was the aim of the present study to define the relative contribution of endogenously generated $\mathrm{NO}$ and $\mathrm{CO}$ to the maintenance of hepatic microvascular flow after HS. We used a rat model of in vivo HS and studied the intrinsic hepatic microvascular response and the associated changes in hepatocellular redox state and secretory function after administration of either the HO inhibitor tin protoporphyrin-IX (SnPP-IX) (22) or the iNOS inhibitor S-methylisothiourea sulfate (SMT) (23) in the isolated perfused liver ex vivo. This methodological approach allows direct in situ visualization of sinusoidal microhemodynamics and analysis of temporal alterations of pyridine nucleotide $(\mathrm{NAD}[\mathrm{P}] \mathrm{H})$ autofluorescence as a marker of reductive stress within individual hepatocytes supplied by these sinusoids and avoids the confounding systemic effects caused by pharmacological interventions. To further characterize the sequence of pathophysiologic events that may ultimately lead to liver dysfunction after HS, we combined these high power epifluorescence microscopic analyses with measurements of total hepatic mitochondrial redox state and bile production.

\section{Methods}

\section{Animals}

Male Sprague-Dawley rats (Charles River, Sulzfeld, Germany), weighing between 300 and $350 \mathrm{~g}$, were used for all experiments. The experimental protocol was approved by the local animal care and use committee, and all animals received humane care according to the criteria outlined in the "Guide for the Care and Use of Laboratory Animals" prepared by the National Academy of Sciences and published by the National Institutes of Health (NIH publication 86-23, revised 1985). Rats were fasted for $6 \mathrm{~h}$ before the induction of anesthesia but allowed free access to water. After anesthesia was induced with an intraperitoneal injection of pentobarbital sodium $(50 \mathrm{mg}$. $\mathrm{kg}^{-1}$ body wt), animals were placed supine on a heating pad (38$40^{\circ} \mathrm{C}$ ) to maintain body temperature. After cannulation of a tail vein an infusion of Ringer's solution $\left(10 \mathrm{ml} \cdot \mathrm{kg}^{-1} \cdot \mathrm{h}^{-1}\right)$ was started to compensate for evaporative losses during the surgical preparation. Anesthesia was maintained with supplemental intravenous (i.v.) bolus injections of pentobarbital sodium $\left(5 \mathrm{mg} \cdot \mathrm{kg}^{-1}\right.$ body wt) when indicated by any evidence of spontaneous muscle activity or lessening of the anesthetic plane. A tracheotomy was performed and animals were allowed to breathe spontaneously. A fluid-filled polyethylene 50 catheter was inserted into the left carotid artery, connected to a pressure transducer (MX 860; Medex Medical Inc., Lancashire, Great Britain), and arterial blood pressure was continuously measured.

\section{Hemorrhagic shock protocol}

HS and resuscitation were performed as previously described (20). After a stabilization period of $10 \mathrm{~min}$, animals in shock groups were rapidly bled to a mean arterial pressure of $40 \mathrm{mmHg}$. Shed blood was collected in syringes containing citrate-phosphate-dextrose solution $\left(0.14 \mathrm{ml} \cdot \mathrm{ml}^{-1}\right.$ of blood; Sigma Chemical Co., St. Louis, MO). Mean arterial pressure was maintained at $40 \pm 4 \mathrm{mmHg}$ by intermittent withdrawal of $0.3-0.4 \mathrm{ml}$ aliquots of blood or infusion of respective aliquots of Ringer's solution. After $60 \mathrm{~min}$ of hemorrhagic hypotension, resuscitation of the animals was performed with $60 \%$ of the shed blood withdrawn (reinfused during the first $20 \mathrm{~min}$ of resuscitation) and twice the maximal bleedout volume as Ringer's solution during the first hour of resuscitation. During the second hour of resuscitation, the infusion rate of Ringer's solution was lowered to a volume equaling the maximal bleedout volume and was subsequently kept constant at $10 \mathrm{ml} \cdot \mathrm{kg}^{-1} \cdot \mathrm{h}^{-1}$ for the remaining resuscitation period. Time-matched sham shock animals were anesthetized and completely instrumented as described above, and received a constant infusion of Ringer's solution of $10 \mathrm{ml} \cdot \mathrm{kg}^{-1} \cdot \mathrm{h}^{-1}$ during the whole observation period but did not undergo hemorrhage (sham shock groups).

\section{Isolated perfused liver system}

A pressure-limited recirculating isolated liver perfusion system was used as previously described $(2,8)$. In brief, the perfusate $(5 \%$ rat red blood cells in Krebs-Henseleit bicarbonate buffer [KHB], $\mathrm{pH} 7.4$ ) was pumped from an outflow reservoir through a Silastic tubing oxygenator (gassed with a mixture of $95 \%$ oxygen $/ 5 \%$ carbon dioxide) into an overflow chamber that served as the inflow reservoir. A heat exchanger was used to warm the perfusate to $37^{\circ} \mathrm{C}$. An ultrasonic in line flow probe (Transonic, Ithaca, NY) was placed ahead of the inlet cannula and connected to a flowmeter (T 206; Transonic) to measure total flow rate $\left(\mathrm{Q}_{\mathrm{t}}\right)$. Portal inlet pressure $\left(\mathrm{P}_{\mathrm{i}}\right)$ was measured via a pressure transducer (Transpac Transducer; Abbott, Wiesbaden, Germany) connected to a T fitting in the inlet cannula. The outflow cannula drained into the outflow reservoir. Another pressure transducer was connected to a second $\mathrm{T}$ fitting in the vena caval outlet cannula to measure outflow pressure $\left(\mathrm{P}_{\mathrm{o}}\right)$. Both pressure transducers were calibrated simultaneously and zeroed against a column of fluid open to the atmosphere at the level of the cannula tips. The two cannulas were positioned at the level of the abdominal posterior vena cava. Signals were recorded using the Windaq 200 personal computerbased data acquisition system (Dataq Instruments, Akron, $\mathrm{OH}$ ). Total portal resistance $\left(\mathrm{R}_{t}\right)$ was calculated from $\left(\mathrm{P}_{\mathrm{i}}-\mathrm{P}_{\mathrm{o}}\right) / \mathrm{Q}_{\mathrm{t}}$.

Allogeneic rat blood was obtained for each experiment from a donor animal after cannulation of the left carotid artery under pentobarbital anesthesia. Red blood cells were separated by centrifugation at 3,000 $\mathrm{g}$ for $3 \mathrm{~min}$. The supernatant (plasma and buffy coat) was discarded. The red blood cell pellet was washed in $0.9 \%$ saline solution, resuspended in an equal volume of KHB, filtered through a Pall PL50 leucocyte removal filter (Pall Newquay, Cornwall, U.K.), and stored at $4^{\circ} \mathrm{C}$ for no longer than $30 \mathrm{~min}$ before use.

\section{Experimental protocol}

On the basis of the time course of shock-induced gene expression of HO-1 and iNOS $(20,24)$, baseline measurements and subsequent blockade of the two mediator systems were performed at $6 \mathrm{~h}$ after shock induction (i.e., $1 \mathrm{~h}$ shock, $5 \mathrm{~h}$ resuscitation; shock groups) or in time-matched sham shock experiments ( $1 \mathrm{~h}$ sham shock, $5 \mathrm{~h}$ sham resuscitation; sham shock groups). At $30 \mathrm{~min}$ before baseline, a laparotomy was performed, the common bile duct was cannulated with a PE 10 catheter, and livers were isolated perfused in situ via the portal vein essentially as previously described $(2,8)$. The initial flow rate was set to $30 \mathrm{ml} \cdot \mathrm{min}^{-1}$ and the perfusate hematocrit was adjusted to $\sim 5 \%$ by addition of rat red blood cells prepared as described above. Fluorescein isothiocyanate-labeled bovine serum albumin was added to the perfusate for assessment of sinusoidal boundaries and visualization of red blood cells in the sinusoids via negative contrast (25). Subsequently, the flow rate was reduced to $20 \mathrm{ml} \cdot \mathrm{min}^{-1}$, and after a 
15-min stabilization period, all baseline measurements were obtained.

Three different series of experiments were conducted. In the first series, a single dose of SnPP-IX (Porphyrin Products, Logan, UT), a potent and specific inhibitor of the CO-generating enzyme $\mathrm{HO}(22)$, was added to the perfusate of livers isolated from either sham shock $(n=7)$ or shock animals $(n=6)$. SnPP-IX was administered at a final concentration of $50 \mu \mathrm{M}$ immediately after all baseline measurements were obtained. In a second experimental series, SMT (Calbiochem, San Diego, CA), a potent and preferential inhibitor of iNOS (23), was added ( $n=6$ per group). SMT was used at a final concentration of $100 \mu \mathrm{M}$, the $\mathrm{EC}_{90}$ value for inhibition of iNOS in macrophages stimulated with bacterial lipopolysaccharide (26). In a third series, 1 $\mathrm{ml}$ of vehicle (KHB) was added to the perfusate of livers from either sham shock or shock animals as a control ( $n=6$ per group). Posttreatment measurements were performed after $10 \mathrm{~min}$, since pilot experiments had shown that the macro- and microhemodynamic effects of SnPP-IX and SMT reached a stable plateau after $5 \mathrm{~min}$ and that this lasted for $\sim 5-10 \mathrm{~min}$.

\section{Epifluorescence liver microscopy}

Analysis of the hepatic microcirculation. Videomicroscopy of the liver was performed essentially as reported in detail previously $(2,8)$. In brief, the liver preparation was positioned on the stage of a Zeiss Axiotech fluorescence microscope (Axiotech Vario $100 \mathrm{HD}$; Carl Zeiss Jena GmbH, Jena, Germany) and viewed with a $40 \times$ water-immersion objective (Zeiss Achroplan; Carl Zeiss Jena GmbH, Jena, Germany). The surface of the left liver lobe was epi-illuminated with a fiber-optic illuminator (KL 1500; Schott Glaswerke, Wiesbaden, Germany) similar to the procedure described by MacPhee et al. (27), and a randomly chosen acinus was brought into focus so that sinusoids of the zone 3 could be observed. This region of the acinus was chosen because the parallel arrangement of sinusoids permits unambiguous quantitative assessment of the sinusoidal microcirculation (28). With the same area in focus, the liver was epi-illuminated with a 100-W mercury lamp for $10 \mathrm{sec}$ with $365-\mathrm{nm}$ excitation and $420-\mathrm{nm}$ emission band-pass filters that allow identification of Ito cells (hepatic stellate cells) by their property as the major storage site of vitamin A, which exhibits bright and rapidly photobleached autofluorescence when excited with light of this wavelength (29). For visualization of fluorescein isothiocyanate-conjugated albumin within hepatic sinusoids, a 450-490 $\mathrm{nm}$ excitation and a 520-nm emission band pass filter (15-sec exposure time) was used. All microscopic images were projected onto a charge-coupled device video camera (FK 6990 IQ-S; Pieper GmbH, Schwerte, Germany). On-line digital contrast enhancement was performed on all images for improved clarity using an Argus-20 image processor (Hamamatsu Photonics K.K., Hamamatsu City, Japan). Processed images were recorded for off-line analysis using an S-VHS video recorder (Panasonic AG 7350E; Matsushita Electrical Industrial Co., Osaka, Japan).

Assessment of sinusoid diameter $\left(D_{S}\right)$ and red blood cell velocity $\left(\mathrm{V}_{\mathrm{RBC}}\right)$ was done off-line during video playback at sites associated with vitamin A autofluorescence and at sinusoidal segments without vitamin A autofluorescence, as we have previously described (29) using digitized frame-by-frame analysis with the Lobulus image analysis system (Medvis, Homburg, Germany) at a specimen-to-monitor ratio of 2,400×. Pre- and posttreatment measurements were performed within the same segments of the same sinusoids. Volumetric flow $\left(\mathrm{Q}_{\mathrm{V}}\right)$ and resistance $\left(\mathrm{R}_{\mathrm{S}}\right)$ in sinusoids were estimated from $\mathrm{V}_{\mathrm{RBC}}$ and the cross-sectional area of the sinusoids according to the equation of Gross and Aroesty (30): $\mathrm{Q}_{\mathrm{V}}=\mathrm{V}_{\mathrm{RBC}} \cdot \pi \cdot \mathrm{r}^{2}\left[\mathrm{pl} \cdot \mathrm{s}^{-1}\right]$ where $\mathrm{r}$ is the sinusoid radius defined as $\mathrm{D}_{\mathrm{S}} / 2(29)$.

Analysis of autofluorescence of pyridine nucleotides. Autofluorescence of reduced pyridine nucleotides $(\mathrm{NAD}[\mathrm{P}] \mathrm{H})$ has been used as a measure of mitochondrial redox state in various tissues (31), including the liver $(32,33)$. Thus, to estimate changes in hepatocellular energy status alterations in $\mathrm{NAD}(\mathrm{P}) \mathrm{H}$ autofluorescence were visually quantified using the same epifluorescence microscopic system (34).
Pre-exposure of the liver surface to ultraviolet epi-illumination for identification of Ito cells at $10 \mathrm{~min}$ before baseline measurements (see above) completely abolished vitamin A-associated autofluorescence, which may otherwise interfere with $\mathrm{NAD}(\mathrm{P}) \mathrm{H}$ autofluorescence (35). Autofluorescence of $\mathrm{NAD}(\mathrm{P}) \mathrm{H}$ was visualized using 365-nm excitation and 420-nm emission band-pass filters. Microscopic images were digitized, stored directly to hard disk, and pixel intensities were measured off-line in the cytoplasm of individual hepatocytes by using the computer-assisted HPD-CP/Argus-20 digital image processing and analysis system (Hamamatsu Photonics K.K., Hamamatsu City, Japan). Pre- and posttreatment measurements were performed within the same hepatocytes. Temporal changes of cytoplasmatic $\mathrm{NAD}(\mathrm{P}) \mathrm{H}$ autofluorescence intensity $\left(\mathrm{I}_{\mathrm{NAD}[\mathrm{P}] \mathrm{H}}\right)$ were calculated according to the equation: $\Delta \mathrm{I}_{\mathrm{NAD}(\mathrm{P}) \mathrm{H}}=$ $\mathrm{I}_{\mathrm{NAD}(\mathrm{P}) \mathrm{H}}$ (posttreatment) $-\mathrm{I}_{\mathrm{NAD}(\mathrm{P}) \mathrm{H}}$ (baseline).

\section{Determination of acetoacetate and $\beta$-hydroxybutyrate perfusate concentrations}

Samples of effluent perfusate were mixed with ice-cold $30 \%$ perchloric acid and centrifuged immediately at $4^{\circ} \mathrm{C}$. The clear supernatant was frozen in liquid nitrogen and stored at $-80^{\circ} \mathrm{C}$ for subsequent analysis. Concentrations of acetoacetate (AcAc) and $\beta$-hydroxybutyrate $(\beta \mathrm{OHB})$ were measured spectrophotometrically as previously described (36). The perfusate ketone body ratio was expressed as AcAc/ $\beta$ OHB to provide an estimate of changes in total hepatic mitochondrial redox state of pyridine nucleotides (37).

\section{Determination of bile production}

Bile was collected during 5-min periods at baseline and $10 \mathrm{~min}$ after pharmacological intervention. Total bile production was assessed by the weight difference of tared microcentrifuge tubes used to collect the bile.

\section{Data analysis}

Data are presented as mean \pm SE (standard error of the mean). Raw data at baseline are provided in Table I. To account for differences in baseline data among the different pretreatment groups and to allow comparison of the response to the subsequent pharmacological interventions, data presented in the figures are expressed as absolute changes from baseline. Statistical differences from baseline within each group were determined using a paired $t$ test. Differences between shock and sham shock groups were tested using an unpaired $t$ test (within each experimental series) or a one-way ANOVA fol-

Table I. Effect of Hemorrhagic Shock on Hepatic Macro- and Microhemodynamics, Mitochondrial Redox State, and Secretory Function in Isolated Perfused Rat Livers at Baseline

\begin{tabular}{lccc}
\hline & Units & Sham shock & Shock \\
\hline $\mathrm{P}_{\mathrm{i}}-\mathrm{P}_{\mathrm{o}}$ & $\mathrm{cmH}_{2} \mathrm{O}$ & $9.4 \pm 0.41$ & $10.0 \pm 0.43$ \\
$\mathrm{Q}_{\mathrm{t}}$ & $\mathrm{ml} \cdot \mathrm{min}^{-1}$ & $20.2 \pm 0.05$ & $20.1 \pm 0.05$ \\
$\mathrm{R}_{\mathrm{t}}$ & $\mathrm{cmH} \mathrm{H}_{2} \mathrm{O} \cdot \mathrm{min} \cdot \mathrm{ml}^{-1}$ & $0.47 \pm 0.021$ & $0.49 \pm 0.021$ \\
$\mathrm{~V}_{\mathrm{RBC}}$ & $\mu \mathrm{m} \cdot \mathrm{s}^{-1}$ & $401 \pm 23.4$ & $274 \pm 24.6^{*}$ \\
$\mathrm{D}_{\mathrm{s}}$ & $\mu \mathrm{m}$ & $12.4 \pm 0.23$ & $12.1 \pm 0.26$ \\
$\mathrm{Q}_{\mathrm{v}}$ & $\mathrm{pl} \cdot \mathrm{s}^{-1}$ & $48.6 \pm 3.07$ & $30.8 \pm 2.77^{*}$ \\
$\mathrm{R}_{\mathrm{s}}$ & $10^{-5} \cdot \mathrm{cmH}_{2} \mathrm{O} \cdot \mathrm{s} \cdot \mathrm{pl}^{-1} \cdot \mu \mathrm{m}^{-1}$ & $2.94 \pm 0.202$ & $3.85 \pm 0.454$ \\
AcAc/BOHB & & $0.59 \pm 0.079$ & $0.45 \pm 0.143^{*}$ \\
Bile flow & $\mathrm{mg} \cdot 5 \mathrm{~min}^{-1}$ & $32.8 \pm 1.77$ & $31.4 \pm 1.12$ \\
& & &
\end{tabular}

Livers were obtained from rats subjected to hemorrhagic shock (1 h; mean arterial pressure: $40 \pm 4 \mathrm{mmHg})$ and volume resuscitation $(5 \mathrm{~h}$; "shock"; $n=18$ experiments) or from time-matched sham shock animals ("sham shock"; $n=19$ experiments), and isolated perfused via the portal vein. After a stabilization period of $15 \mathrm{~min}$, all baseline measurements were obtained. Values are mean \pm SE. $* P<0.05$ vs. sham shock. 
lowed by post-hoc Student-Newman-Keuls tests for pairwise comparisons (between groups of different experimental series). When criteria for parametric tests were not met (Kolomogorov-Smirnow Test for normality and/or Levene-Mediane test for equal variance failed), the respective nonparametric tests (i.e., Wilcoxon signed rank test, Mann-Whitney rank sum test and Kruskal-Wallis ANOVA on ranks followed by Dunn's test) were used. Correlation analysis between changes in $\mathrm{NAD}(\mathrm{P}) \mathrm{H}$ autofluorescence intensity in individual hepatocytes and changes in mean $\mathrm{Q}_{\mathrm{V}}$ in the two adjacent sinusoids was performed using the Pearson-product moment test. A $P$ value $<0.05$ was considered to indicate a significant difference. All statistical tests were performed using the SigmaStat software package (Jandel Scientific, San Rafael, CA).

\section{Results}

Effect of hemorrhagic shock on baseline hepatic macro- and micro-hemodynamics, mitochondrial redox state, and bile production. HS had no effect on baseline hepatic macrohemodynamics in isolated perfused livers, since $P_{i}-P_{o}, Q_{t}$, and $R_{t}$ were similar after HS compared with sham shock (Table I). However, epifluorescence microscopy of the liver revealed shock-induced microcirculatory disturbances. Both, $\mathrm{V}_{\mathrm{RBC}}$ and $Q_{V}$ were lower after HS compared with sham shock, while $D_{S}$ and $R_{S}$ were not different (Table I). HS resulted in a moderate shift of the mitochondrial redox potential to a more reduced state, since AcAc/ $\mathrm{BOHB}$ was lower after HS compared with sham shock, but this did not affect bile production (Table I).

Effect of SnPP-IX and SMT on hepatic macrohemodynamics. While vehicle administration had no significant macrohemodynamic effects, addition of SnPP-IX to the perfusate of sham shock livers caused an increase in $P_{i}-P_{o}$ and a decrease in $Q_{t}$ that was associated with a moderate increase in $R_{t}$ compared with baseline (Fig. 1). However, the increase in $R_{t}$ and the respective changes in $P_{i}-P_{o}$ and $Q_{t}$ were much more pronounced in shock livers (Fig. 1). In contrast, addition of SMT to the perfusate of sham shock livers only resulted in a small decrease in $Q_{t}$ and did not affect $P_{i}-P_{o}$ and $R_{t}$ under these conditions. Although the decrease in $\mathrm{Q}_{t}$ after HS and SMT administration was slightly greater compared with the change in $Q_{t}$ after sham shock and SMT, the associated increase in $R_{t}$ (Fig. 1) was much lower than the increase in $R_{t}$ that occurred after SnPP-IX administration to shock livers $(0.08 \pm 0.025$ vs. $\left.1.38 \pm 0.338 \mathrm{cmH}_{2} \mathrm{O} \cdot \min \cdot \mathrm{ml}^{-1} ; P<0.01\right)$. Furthermore, triplicating the SMT dose, i.e., administration of $3 \times \mathrm{EC}_{90}$ for SMT-mediated inhibition of iNOS (26), did not result in a greater increase in $\mathrm{R}_{\mathrm{t}}$ compared with addition of $1 \times \mathrm{EC}_{90}$ (data not shown).

Effect of SnPP-IX and SMT on hepatic microhemodynamics. Vehicle administration to sham shock or shock livers did not cause any significant microcirculatory changes (Fig. 2). Addition of SnPP-IX to the perfusate of livers from sham shock animals decreased $\mathrm{V}_{\mathrm{RBC}}$ and $\mathrm{Q}_{\mathrm{V}}$ and caused a small increase in $\mathrm{R}_{\mathrm{S}}$ compared with baseline (Fig. 2). After HS, the increase in $R_{S}$ and the decrease in $V_{R B C}$ and $Q_{V}$ induced by SnPP-IX was much more pronounced compared with sham shock (Fig. 2). In sharp contrast, administration of SMT did not affect hepatic microhemodynamics of sham shock livers and caused only a slight decrease in $\mathrm{V}_{\mathrm{RBC}}$ in the shock group (Fig. 2).

While neither vehicle nor SMT administration had any significant effects on $\mathrm{D}_{S}$ in sham shock or shock groups, SnPP-IX caused a decrease in $\mathrm{D}_{\mathrm{S}}$ at sites corresponding to vitamin $\mathrm{A}$


Figure 1. Effect of hemorrhagic shock on changes in portal driving pressure (inlet-outlet pressure; $A$ ), total portal flow rate $(B)$, and total portal vascular resistance $(C)$ in isolated perfused rat livers in response to either vehicle ( $1 \mathrm{ml} \mathrm{KHB})$, or SnPP-IX, a specific inhibitor of the CO-generating enzyme heme oxygenase $(50 \mu \mathrm{M})$, or SMT, a preferential inhibitor of the inducible NO synthase $(100 \mu \mathrm{M})$. Posttreatment measurements were performed 10 min after pharmacological intervention. Livers were obtained from rats subjected to hemorrhagic shock ( $1 \mathrm{~h}$; mean arterial pressure: $40 \pm 4 \mathrm{mmHg}$ ) and volume resuscitation ( $5 \mathrm{~h}$; shock groups; filled bars) or from time-matched sham shock animals (open bars). Data represent mean \pm SE for $n=7$ experiments (sham shock/SnPP-IX group), and $n=6$ experiments (all other groups). ${ }^{*}, P<0.05$ vs. respective baseline value (within each group); $\#, P<0.05$ vs. respective sham shock group (within each series).

fluorescence in the sham shock group (Fig. 3). The sinusoidal constriction in response to SnPP-IX apparently occurred specifically at these sinusoidal segments. While $\mathrm{D}_{\mathrm{S}}$ did not show any significant changes in response to SnPP-IX at sites without 


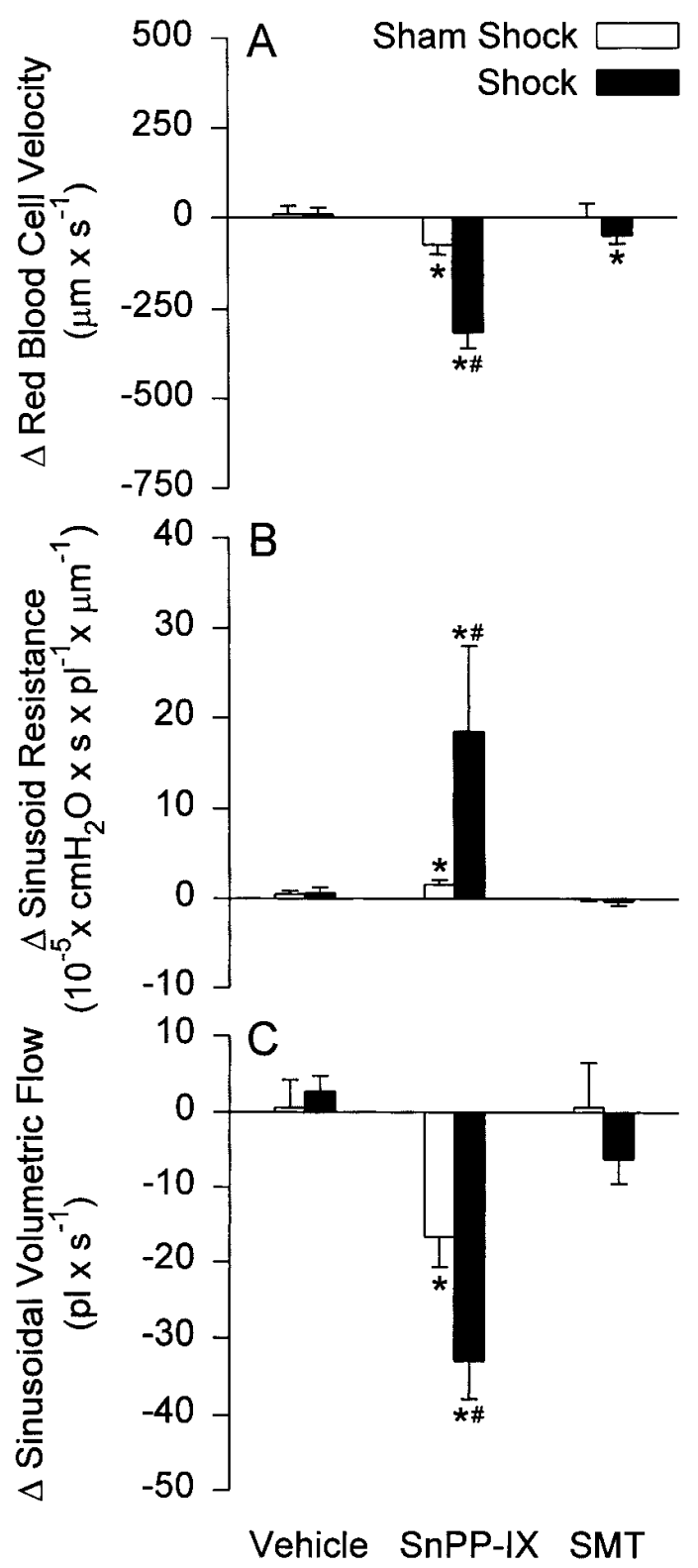

Figure 2. Effect of hemorrhagic shock on changes in red blood cell velocity in sinusoids $(A)$, sinusoid resistance $(B)$, and sinusoidal volumetric flow $(C)$ in isolated perfused rat livers in response to either vehicle or SnPP-IX $(50 \mu \mathrm{M})$ or SMT $(100 \mu \mathrm{M})$. Posttreatment measurements were performed $10 \mathrm{~min}$ after pharmacological intervention. Livers were obtained from rats subjected to hemorrhagic shock (shock groups; filled bars) or from time-matched sham shock animals (open bars). Red blood cell velocity in sinusoids and sinusoid diameters (compare Fig. 3) were determined using in situ epifluorescence videomicroscopy, and resistance and volumetric flow in sinusoids were calculated from red blood cell velocity and cross-sectional area of the sinusoid. Data represent mean \pm SE for $n=7$ experiments (sham shock/SnPP-IX group) and $n=6$ experiments (all other groups). *, $P<0.05$ vs. respective baseline value (within each group); $\#, P<0.05$ vs. respective sham shock group (within each series).

vitamin A autofluorescence, the sinusoidal width measured at sites of autofluorescence following SnPP-IX decreased by $\sim 15 \%$ (Fig. 3). After HS, the sinusoidal narrowing that occurred at vitamin A autofluorescence sites in response to



Figure 3. Effect of hemorrhagic shock on changes in sinusoid diameter in isolated perfused rat livers in response to either vehicle ( $A$ and $B$ ), or SnPP-IX (50 $\mu \mathrm{M} ; C$ and $D)$, or SMT $(100 \mu \mathrm{M} ; E$ and $F)$. Posttreatment measurements were performed $10 \mathrm{~min}$ after pharmacological intervention. Livers were obtained from rats subjected to hemorrhagic shock (shock groups; closed symbols) or from time-matched sham shock animals (open symbols). Sinusoid diameters were determined at sites associated with vitamin A autofluorescence (vit. A AF; triangles) and at sinusoidal segments without vitamin A autofluorescence (no vit. A AF; circles) using in situ epifluorescence videomicroscopy. Data represent mean \pm SE for $n=7$ experiments (sham shock/SnPP-IX group) and $n=6$ experiments (all other groups). *, $P<0.05$ vs. respective baseline value (within each group); ${ }^{\S}, P<0.05$ vs. respective sites without vitamin A autofluorescence (within each group); $\#, P<0.05$ vs. respective sinusoidal segments of the sham shock group (within each series). 
SnPP-IX was more pronounced compared with sham shock $(-3.2 \pm 0.39$ vs. $-1.7 \pm 0.27 \mu \mathrm{m} ; P<0.01)$. Furthermore, after $\mathrm{HS}$, the SnPP-IX-mediated reduction in $\mathrm{D}_{\mathrm{S}}$ was no longer confined to these sinusoidal segments but could also be observed at sites of the hepatic sinusoid not associated with vitamin A autofluorescence (Fig. 3). However, the decrease in sinusoidal width at autofluorescence sites of shock livers after SnPP-IX administration was still much greater than at sites without vitamin A autofluorescence (Fig. 3).

Effect of SnPP-IX and SMT on hepatic mitochondrial redox state and bile production. To survey the effect of the pharmacological interventions on hepatic mitochondrial redox state, two independent methods were applied. First, changes in $\mathrm{NAD}(\mathrm{P}) \mathrm{H}$ fluorescence intensity were analyzed within individual hepatocytes using epifluorescence microscopy. Microscopic images from three typical experiments are shown in Fig. 4. Quantitative results of estimations of changes in $\mathrm{NAD}(\mathrm{P}) \mathrm{H}$ fluorescence intensity from all experiments are depicted in Fig. 5. A profound increase in $\mathrm{NAD}(\mathrm{P}) \mathrm{H}$ autofluorescence was observed after SnPP-IX in livers from animals after HS (Fig. 4, $C$ and $D$; Fig. 5). Furthermore, there was a significant negative correlation between the changes in $\mathrm{NAD}(\mathrm{P}) \mathrm{H}$ autofluorescence intensity in individual hepatocytes and the changes in mean $\mathrm{Q}_{\mathrm{V}}$ in the two adjacent sinusoids $(r=-0.58, P=0.01)$. In sharp contrast, under all other experimental conditions, $\mathrm{NAD}(\mathrm{P}) \mathrm{H}$ fluorescence intensity either remained unchanged or decreased slightly (Fig. 4, $A$ and $B, E$ and $F$; Fig. 5). As a second independent assessment of hepatocellular energy charge, the AcAc and $\beta \mathrm{OHB}$ concentrations in the effluent perfusate were determined. Thus, it is of particular interest that the AcAc/ $\beta \mathrm{OHB}$ ratio revealed similar changes in total hepatic mitochondrial redox state. A shift in the redox potential to a more reduced state, i.e., a decrease in $\mathrm{AcAc} / \beta \mathrm{OHB}$, could only be observed after HS and subsequent SnPP-IX administration, whereas under all other experimental conditions, the $\mathrm{AcAc} / \beta \mathrm{OHB}$ either remained unchanged or increased moderately (Fig. 5). Furthermore, this impairment in hepatic mitochondrial energy charge after HS and SnPP-IX was asso- ciated with a decrease in bile production by $\sim 50 \%$, while hepatic secretory function remained unchanged under all other conditions studied (Fig. 6).

\section{Discussion}

In the present study, we investigated the differential role of the two gaseous monoxides $\mathrm{NO}$ and $\mathrm{CO}$ in the intrinsic hepatic regulation of microvascular flow after HS. Our results demonstrate for the first time that endogenously generated $\mathrm{CO}$ acts to maintain sinusoidal perfusion, mitochondrial redox state, and secretory function of the liver after HS. In contrast, the alternate stress-inducible guanylate cyclase activating pathway, i.e., the generation of NO by the inducible isoform of NOS, seems to be of only minor importance for the control of hepatic perfusion and function under these conditions.

In agreement with earlier studies $(38,39)$, HS caused hepatic microcirculatory disturbances, i.e., resulted in a reduced $\mathrm{V}_{\mathrm{RBC}}$ and $\mathrm{Q}_{\mathrm{V}}$. However, $\mathrm{D}_{\mathrm{S}}$ was similar after $\mathrm{HS}$ compared with sham shock, and the shock-induced reductions in sinusoidal flow were only moderate, despite the fact that ischemia/ reperfusion of the liver has been shown to result in increased hepatic expression of endothelin-1 (40), a potent vasoconstrictor in the hepatic microcirculation $(29,41)$. This suggests that a simultaneous induction of vasodilatory mediators may serve a protective role in hepatic microcirculatory dysfunction after HS. Previous studies have shown that $\mathrm{CO}$ can activate soluble guanylate cyclase (42), which in turn causes a rise in intracellular cyclic guanosine $3^{\prime}, 5^{\prime}$-monophosphate concentrations that leads to smooth muscle relaxation in aortic rings (14). However, the vasodilatory effect of $\mathrm{CO}$ is not confined to the aorta but also can be demonstrated in other vascular beds, including the hepatic portal venous macro- and microcirculation (1618). Moreover, the liver is one of the most abundant sources of HO activity among organs under physiological conditions (13). Since HS results in enhanced hepatic expression of HO-1, the stress-inducible isoform of the CO-generating enzymes (20, 21), we hypothesized that $\mathrm{CO}$ could act to maintain hepatic si-

\section{Shock/SnPP-IX}

\section{Shock/SMT}

\section{Baseline Posttreatment}

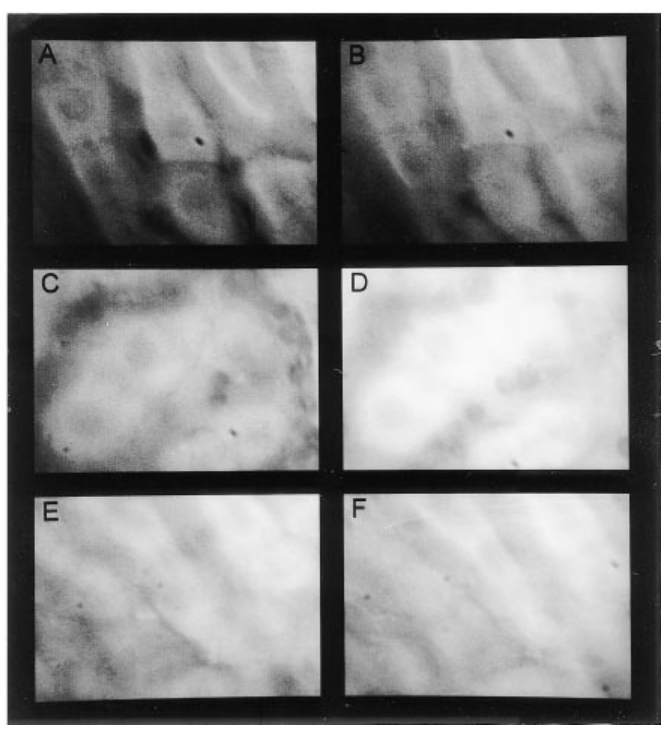

Figure 4. Effect of hemorrhagic shock on changes in cytoplasmatic autofluorescence intensity of reduced pyridine nucleotides $(\mathrm{NAD}[\mathrm{P}] \mathrm{H})$ of hepatocytes in response to either vehicle or SnPP-IX $(50 \mu \mathrm{M})$ or SMT $(100 \mu \mathrm{M})$. Posttreatment measurements were performed 10 min after pharmacological intervention. Livers were obtained from rats subjected to hemorrhagic shock or from a time-matched sham shock animal. NAD(P)H autofluorescence was visualized using in situ highpower microscopy. Images from three typical experiments are shown (original magnification: $\times 2,400)$. 


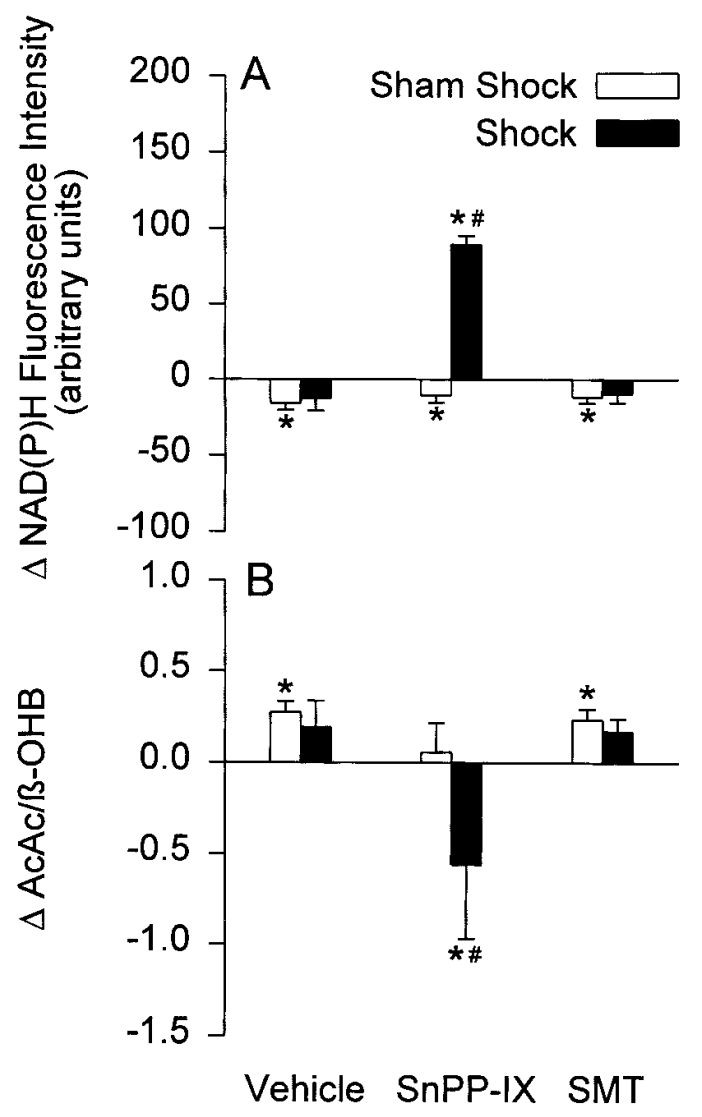

Figure 5. Effect of hemorrhagic shock on changes in cytoplasmatic autofluorescence intensity of $\mathrm{NAD}(\mathrm{P}) \mathrm{H}$ of hepatocytes $(A)$ and $\mathrm{AcAc} / \beta \mathrm{OHB}$ in the effluent perfusate $(B)$ in response to either vehicle or SnPP-IX $(50 \mu \mathrm{M})$ or SMT $(100 \mu \mathrm{M})$. Posttreatment measurements were performed $10 \mathrm{~min}$ after pharmacological intervention. Livers were obtained from rats subjected to hemorrhagic shock (shock groups; filled bars) or from time-matched sham shock animals (open bars). $\mathrm{NAD}(\mathrm{P}) \mathrm{H}$ autofluorescence was visualized using in situ high-power microscopy, and changes in fluorescence intensity were measured off-line using a digital image analysis system. Concentrations of $\mathrm{AcAc}$ and $\beta \mathrm{OHB}$ were determined spectrophotometrically and the $\mathrm{Ac} A \mathrm{~A} / \mathrm{\beta OHB}$ ratio was calculated to provide an estimate of changes in total hepatic mitochondrial redox state of pyridine nucleotides. Data represent mean \pm SE for $n=7$ experiments (sham shock/ SnPP-IX group) and $n=6$ experiments (all other groups). *, $P<0.05$ vs. respective baseline value (within each group); \#, $P<0.05$ vs. respective sham shock group (within each series).

nusoidal perfusion after HS. Here, we show that administration of the HO inhibitor SnPP-IX after HS caused increases in total portal and sinusoid resistance and respective decreases in sinusoidal volumetric flow that were much more pronounced than in sham shock experiments. These findings strongly suggest that endogenous generation of the vasodilator $\mathrm{CO}$ serves a protective role in hepatic microcirculatory dysfunction after HS, i.e., limits the extent of shock-induced sinusoidal perfusion failure caused by the simultaneous upregulation of vasoconstrictive mediators in the liver such as endothelins $(40,43)$.

One aim of this study was to characterize the microvascular mechanisms through which $\mathrm{CO}$ modulates sinusoid resistance after HS. Because of the absence of vascular smooth muscle in

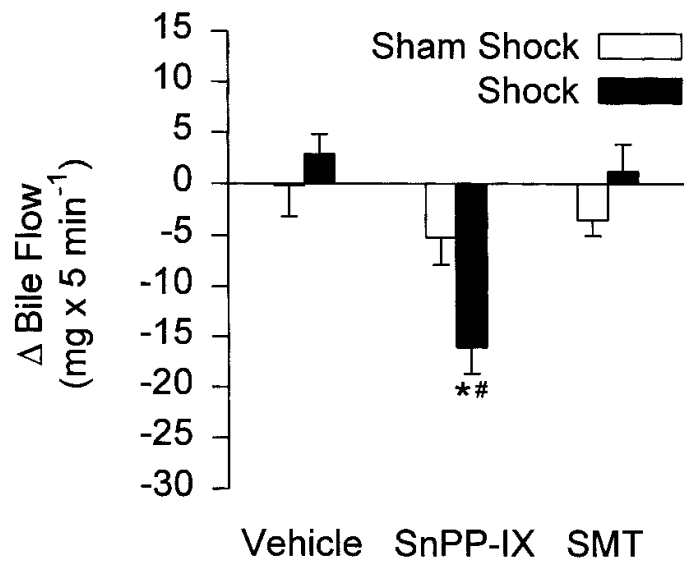

Figure 6. Effect of hemorrhagic shock on changes in bile flow rate in isolated perfused rat livers in response to either vehicle or SnPP-IX $(50 \mu \mathrm{M})$ or SMT $(100 \mu \mathrm{M})$. Posttreatment measurements were performed $10 \mathrm{~min}$ after pharmacological intervention. Livers were obtained from rats subjected to hemorrhagic shock (shock groups; filled bars) or from time-matched sham shock animals (open bars). Data represent mean \pm SE for $n=7$ experiments (sham shock/SnPP-IX group) and $n=6$ experiments (all other groups). *, $P<0.05$ vs. respective baseline value (within each group); \#, $P<0.05$ vs. respective sham shock group (within each series).

the sinusoids, other cell types must be implicated in causing changes in sinusoid diameter. Recent studies have suggested that the control of sinusoidal tone is mediated, at least in part, by Ito cells (44). These cells are localized in the space of Dissé and have several anatomical and functional similarities to vascular pericytes (45). Moreover, we have previously shown that after administration of exogenous endothelin-1, sinusoidal constriction colocalized with Ito cell-associated autofluorescence of vitamin A $(29,46)$. Therefore, we analyzed the changes in sinusoid diameter upon administration of SnPP-IX at sites associated with vitamin A autofluorescence and compared them with the changes in $D_{s}$ at those segments of the sinusoids that were not associated with vitamin A autofluorescence. In the livers isolated from sham shock animals, the sinusoidal constriction in response to SnPP-IX occurred specifically at sites associated with vitamin A autofluorescence, i.e., at the location of the peri-nuclear cytoplasm of Ito cells, which is in agreement with the results of previous reports by Suematsu et al. $(17,18)$. However, despite the fact that in response to SnPP-IX the sinusoidal narrowing at autofluorescence sites was more pronounced after HS compared with sham shock, the reduction in $\mathrm{D}_{\mathrm{s}}$ induced by inhibition of the $\mathrm{HO}-\mathrm{CO}$ pathway was no longer confined to these sinusoidal segments following HS. Rather, a discrete narrowing also could be observed at other segments of the hepatic sinusoid not associated with vitamin A autofluorescence. The following factors may have contributed to these findings. On one hand, these results would be consistent with the notion that relaxation of hepatic Ito cells mediates, at least in part, the flow-enhancing effect of $\mathrm{CO}$ in the hepatic microcirculation after HS and the sinusoidal narrowing at segments not associated with vitamin A autofluorescence may be due to the fact that Ito cell contraction affects most likely the configuration of other neighboring components of the sinusoidal wall, in particular because the numerous pro- 
cesses of Ito cells reach out to wrap around the sinusoid far beyond the peri-nuclear region containing the vitamin A droplets (47). Alternatively, these findings may reflect that, in contrast to the normal liver, $\mathrm{CO}$ exerts its relaxing effects after HS not only on Ito cells but also to a lesser degree on other cells of the sinusoidal wall. In this regard, it is of particular interest that in addition to Ito cells, Kupffer cells as well as sinusoidal endothelial cells have been shown to contain filaments, tubules, and contractile proteins suggestive of contractile activity, and changes in tone of all three cell types in response to a wide variety of pharmacodynamic substances have been reported (48). Furthermore, it is important to note that sinusoid resistance is only one component of total portal resistance (49), and extrasinusoidal mechanisms, e.g., constriction of portal venules (50), may also have profound effects on sinusoidal flow and diameter (41). Therefore, additional studies will be needed that further characterize the relative contribution of sinusoidal and extrasinusoidal sites of CO-mediated vasodilation to the protection of the hepatic microcirculation after HS.

The following findings suggest that the sinusoidal flowenhancing effects of $\mathrm{CO}$ after HS may be important for the limitation of shock-induced liver dysfunction. First, the substantial reductions in sinusoidal flow that occurred upon SnPPIX administration after HS were associated with a dramatic shift in mitochondrial redox potential to a more reduced state that could be demonstrated using two independent methods. This is of particular importance since a recent study by Nakatani et al. (51) revealed a significant inverse relationship between the impairment of hepatic mitochondrial redox state and the survival rate after HS in humans. Second, the perturbations of the hepatic microcirculation and energy charge after HS and SnPP-IX were associated with a decrease in total bile production, an estimate of hepatocellular secretory function. Third, the existence of a functional link between CO-mediated protection of the hepatic microcirculation and the development of hepatocellular dysfunction after HS is further supported by the fact that correlation analysis revealed a significant inverse relationship between changes in $\mathrm{NAD}(\mathrm{P}) \mathrm{H}$ autofluorescence intensity within individual hepatocytes and microvascular flow within the adjacent sinusoids supplying this specific parenchymal cell. Furthermore, the protective effects of $\mathrm{CO}$ do not seem to be confined to the isolated perfused liver preparation used in the present study, because blockade of $\mathrm{CO}$ production after HS in rats in vivo increased the release of $\alpha$-glutathione-S-transferase from the liver and resulted in a higher incidence of pericentral hepatic necrosis compared to HS alone (H. Rensing, unpublished observation).

Previous studies from our and other laboratories have provided substantial evidence, that the endothelium-dependent vasodilator NO, generated by the constitutive isoform of NOS (cNOS), acts to preserve hepatic blood flow early ( $\leq 1 \mathrm{~h}$ ) after low-flow ischemia/reperfusion of the isolated perfused liver (52) and after resuscitation from HS in vivo (43), thereby limiting hepatocyte injury (53). However, while de novo expression of iNOS in the liver has been shown to occur at $\geq 5 \mathrm{~h}$ after exposure to bacterial endotoxin (24), and increased hepatic generation of $\mathrm{NO}$ by iNOS has been shown to minimize microcirculatory dysfunction in the liver after endotoxemic shock (8), the functional role of iNOS-derived NO in the control of sinusoidal perfusion after HS is unknown. In the present study, administration of the preferential iNOS inhibitor SMT after HS caused only a small increase in $\mathrm{R}_{\mathrm{t}}$ that was associated with a minor decrease in $\mathrm{V}_{\mathrm{RBC}}$ and had no effect on sinusoidal resistance or flow. Furthermore, SMT caused no deteriorations of hepatic mitochondrial redox state or bile flow. These findings strongly suggest that NO, generated by the inducible isoform of NOS, is not an important factor in the control of hepatic microvascular flow, energy state, and bile production after HS, which is internally consistent with a report by Kelly et al. (54) showing that in contrast to endotoxemic shock, there was no significant hepatic induction of iNOS after HS in rats in vivo neither on the mRNA nor on the protein level.

In conclusion, endogenously generated $\mathrm{CO}$ acts to preserve sinusoidal perfusion, mitochondrial redox state, and secretory function in the isolated perfused rat liver after HS. This protective role of $\mathrm{CO}$ in hepatic microcirculatory dysfunction after HS is mediated via a relaxing mechanism in part involving Ito cells. In sharp contrast, the alternate stress-inducible guanylate cyclase activating pathway, i.e., the generation of NO by the inducible isoform of NOS, seems to be of only minor importance for the control of hepatic perfusion and function under these conditions. Along with our previous results, these findings suggest that $\mathrm{CO}$ may exert a similar protective function in the hepatic microcirculation after HS, as does NO after endotoxemic shock.

\section{Acknowledgments}

We thank Thomas Berger for expert technical assistance in performing the spectrophotometrical measurements of acetoacetate and $\beta$-hydroxybutyrate, and Dr. Heike Pahl for critical review of the manuscript.

This work was supported by the Deutsche Forschungsgemeinschaft (PA 533/2-1 to B.H.J. Pannen and BA 1601/1-1 to M. Bauer) and the National Institutes of Health (DK38201 to M.G. Clemens).

\section{References}

1. Bauer, I., M. Bauer, B.H.J. Pannen, M.J. Leinwand, J.X. Zhang, and M.G. Clemens. 1995. Chronic ethanol consumption exacerbates liver injury following hemorrhagic shock: role of sinusoidal perfusion failure. Shock. 4:324331.

2. Chun, K., J. Zhang, J. Biewer, D. Ferguson, and M.G. Clemens. 1994. Microcirculatory failure determines lethal hepatocyte injury in ischemic/reperfused rat livers. Shock. 1:3-9.

3. Pannen, B.H.J., and J.L. Robotham. 1995. The acute phase response. New Horiz. 3:183-197.

4. Palmer, R.M.J., A.G. Ferrige, and S. Moncada. 1987. Nitric oxide release accounts for the biological activity of endothelium-derived relaxing factor. $\mathrm{Na}$ ture. 327:524-526.

5. Moncada, S., and A. Higgs. 1993. The L-arginine nitric oxide pathway. $N$. Engl. J. Med. 329:2002-2012.

6. Ayuse, T., N. Brienza, J.P. Revelly, J.K. Boitnott, and J.L. Robotham. 1995. Role of nitric oxide in the porcine liver circulation under normal and endotoxemic conditions. J. Appl. Physiol. 78:1319-1329.

7. Nishida, J., R.S. McCuskey, D. McDonnell, and E.S. Fox. 1994. Protective role of $\mathrm{NO}$ in hepatic microcirculatory dysfunction during endotoxemia. Am. J. Physiol. 267:G1135-G1141.

8. Pannen, B.H.J., M. Bauer, J.X. Zhang, J.L. Robotham, and M.G. Clemens. 1996. A time-dependent balance between endothelins and nitric oxide regulating portal resistance after endotoxin. Am. J. Physiol. 271:H1953-H1961.

9. Geller, D.A., C.J. Lowenstein, R.A. Shapiro, A.K. Nussler, M. DiSilvio, S.C. Wang, D.K. Nakayama, R.L. Simmons, S.H. Snyder, and T.R. Billiar. 1993. Molecular cloning and expression of inducible nitric oxide synthase from human hepatocytes. Proc. Natl. Acad. Sci. USA. 90:3491-3495.

10. Thiemermann, C., C. Szabo, J.A. Mitchell, and J.R. Vane. 1993. Vascular hyporeactivity to vasoconstrictor agents and hemodynamic decompensation in hemorrhagic shock is mediated by nitric oxide. Proc. Natl. Acad. Sci. USA. 90:267-271.

11. Wang, P., Z.F. Ba, and I.H. Chaudry. 1993. Endothelial cell dysfunction occurs very early following trauma-hemorrhage and persists despite fluid resuscitation. Am. J. Physiol. 265:H973-H979.

12. Jacob, T.D., J.B. Ochoa, A.O. Udekwu, J. Wilkinson, T. Murray, T.R. 
Billiar, R.L. Simmons, D.W. Marion, and A.B. Peitzman. 1993. Nitric oxide production is inhibited in trauma patients. J. Trauma. 35:590-597.

13. Maines, M.D. 1988. Heme oxygenase: function, multiplicity, regulatory mechanisms, and clinical implications. FASEB J. 2:2557-2568.

14. Furchgott, R.F., and D. Jothianandan. 1991. Endothelium-dependent and -independent vasodilation involving cyclic GMP: relaxation by nitric oxide, carbon monoxide and light. Blood Vessels. 28:52-61.

15. Morita, T., M.A. Perella, M.-E. Lee, and S. Kourembanas. 1995. Smooth muscle cell-derived carbon monoxide is a regulator of vascular cGMP. Proc. Natl. Acad. Sci. USA. 92:1475-1479.

16. Suematsu, M., S. Kashiwagi, T. Sano, and N. Goda. 1994. Carbon monoxide as an endogenous mediator of hepatic vascular perfusion. Biochem. Biophys. Res. Commun. 205:1333-1337.

17. Suematsu, M., N. Goda, T. Sano, S. Kashiwagnagi, T. Egawa, Y. Shinoda, and Y. Ishimura. 1995. Carbon monoxide: an endogenous modulator of sinusoidal tone in the perfused rat liver. J. Clin. Invest. 96:2431-2437.

18. Goda, N., K. Suzuki, M. Naito, S. Takeoka, E. Tsuchida, Y. Ishimura, T. Tamatani, and M. Suematsu. 1998. Distribution of heme oxygenase isoforms in rat liver. Topographic basis for carbon monoxide-mediated microvascular relaxation. J. Clin. Invest. 101:604-612.

19. McCuskey, R.S. 1995. Does a toxic gas regulate sinusoidal blood flow? J. Clin. Invest. 96:2099.

20. Bauer, M., B.H.J. Pannen, I. Bauer, C. Herzog, G.A. Wanner, R. Hanselmann, J.X. Zhang, M.G. Clemens, and R. Larsen. 1996. Evidence for a functional link between stress response and vascular control in hepatic portal circulation. Am. J. Physiol. 271:G929-G935.

21. Bauer, I., G.A. Wanner, H. Rensing, C. Alte, E.A. Miescher, B. Wolf, B.H.J. Pannen, M.G. Clemens, and M. Bauer. 1998. Expression pattern of heme oxygenase isoenzymes 1 and 2 in normal and stress-exposed rat liver. Hepatology. 27:829-838.

22. Yoshinaga, T., S. Sassa, and A. Kappas. 1982. Purification and properties of bovine spleen heme oxygenase. Amino acid composition and sites of action of inhibitors of heme oxygenase. J. Biol. Chem. 257:7778-7785.

23. Szabo, C., G.J. Southan, and C. Thiemermann. 1994. Beneficial effects and improved survival in rodent models of septic shock with S-methylisothiourea sulfate, a potent and selective inhibitor of inducible nitric oxide synthase. Proc. Natl. Acad. Sci. USA. 91:12472-12476.

24. Cook, H.T., A.J. Bune, A.S. Jansen, G.M. Taylor, R.K. Loi, and V. Cattell. 1994. Cellular localization of inducible nitric oxide synthase in experimental endotoxic shock in the rat. Clin. Sci. 87:179-186.

25. McDonagh, P.F., and S.K. Williams. 1984. The preparation and use of fluorescent-protein conjugates for microvascular research. Microvasc. Res. 27: $17-27$

26. Southan, G.J., C. Szabo, and C. Thiemermann. 1995. Isothioureas: potent inhibitors of nitric oxide synthases with variable isoform selectivity. Br. J. Pharmacol. 114:510-516.

27. MacPhee, P.J., E.E. Schmidt, and A.C. Groom. 1992. Evidence for Kupffer cell migration along liver sinusoids from high-resolution in vivo microscopy. Am. J. Physiol. 263:G17-G23.

28. Clemens, M.G., P.F. McDonagh, I.H. Chaudry, and A.E. Baue. 1985. Hepatic microcirculatory failure after ischemia and reperfusion: improvement with ATP- $\mathrm{MgCl}_{2}$ treatment. Am. J. Physiol. 248:H804-H811.

29. Zhang, J.X., W. Pegoli, and M.G. Clemens. 1994. Endothelin-1 induces direct constriction of hepatic sinusoids. Am. J. Physiol. 266:G624-G632.

30. Gross, J.F., and J. Aroesty. 1972. Mathematical models of capillary flow: a critical review. Biorheology. 9:225-264.

31. Chance, B., P. Cohen, F. Jobsis, and B. Schoener. 1962. Intracellular oxidation-reduction states in vivo. Science. 137:499-508.

32. Scholz, R., R.G. Thurman, J.R. Williamson, B. Chance, and T. Bücher. 1969. Flavin and pyridine nucleotide oxidation-reduction changes in perfused rat liver. J. Biol. Chem. 244:2317-2324.

33. Horie, Y., R. Wolf, D.C. Anderson, and D.N. Granger. 1997. Hepatic leukostasis and hypoxic stress in adhesion molecule-deficient mice after gut ischemia/reperfusion. J. Clin. Invest. 99:781-788.

34. Suzuki, H., M. Suematsu, H. Ishii, S. Kato, H. Miki, M. Mori, Y. Ish- imura, T. Nishino, and M. Tsuchiya. 1994. Prostaglandin $\mathrm{E}_{1}$ abrogates early reductive stress and zone-specific paradoxical oxidative injury in hypoperfused rat liver. J. Clin. Invest. 93:155-164.

35. Suematsu, M., M. Oda, H. Suzuki, H. Kaneko, T. Furusho, S. Masushige, and M. Tsuchiya. 1993. Intravital and electron microscopic observation of Ito cells in rat hepatic microcirculation. Microvasc. Res. 46:28-42.

36. Bergmeyer, H.U. 1974. Methods of Enzymatic Analysis. Academic Press, New York. 1836 pp.

37. Ozawa, K., B. Chance, A. Tanaka, S. Iwata, T. Kitai, and I. Ikai. 1992. Linear correlation between acetoacetate/ $\beta$-hydroxybutyrate in arterial blood and oxidized flavoprotein/reduced pyridine nucleotide in freeze-trapped human liver tissue. Biochim. Biophys. Acta. 1138:350-352.

38. Koo, A., and I.Y.S. Liang. 1977. Blood flow in hepatic sinusoids in experimental hemorrhagic shock in rats. Microvasc. Res. 13:315-325.

39. Bauer, M. I. Marzi, T. Ziegenfuss, G. Seek, V. Buehren, and R. Larsen. 1993. Comparative effects of crystalloid and small volume hypertonic hyperoncotic fluid resuscitation on hepatic microcirculation after hemorrhagic shock. Circ. Shock. 40:187-193.

40. Kawamura, E., N. Yamakana, E. Okamoto, F. Tomoda, and K. Furukawa. 1995. Response of plasma and tissue endothelin-1 to liver ischemia and its implication in ischemia-reperfusion injury. Hepatology. 21:1138-1143.

41. Bauer, M., J.X. Zhang, I. Bauer, and M.G. Clemens. 1994. Endothelin-1 induced alterations of hepatic microcirculation: sinusoidal and extrasinusoidal sites of action. Am. J. Physiol. 267:G143-G149.

42. Kharitonov, V.G., V.S. Sharma, R.B. Pilz, D. Magde, and D. Koesling. 1995. Basis of guanylate cyclase activation by carbon monoxide. Proc. Natl. Acad. Sci. USA. 92:2568-2571.

43. Pannen, B.H.J., M. Bauer, G.F.E. Nöldge-Schomburg, J.X. Zhang, J.L. Robotham, M.G. Clemens, and K.K. Geiger. 1997. Regulation of hepatic blood flow during resuscitation from hemorrhagic shock: role of NO and endothelins. Am. J. Physiol. 272:H2736-H2745.

44. Pinzani, M., P. Failli, C. Ruocco, A. Casini, S. Milani, E. Baldi, A. Giotti, and P. Gentilini. 1992. Fat-storing cells as liver-specific pericytes. Spatia dynamics of agonist-stimulated intracellular calcium transients. J. Clin. Invest. 90:642-646.

45. Rockey, D. 1997. The cellular pathogenesis of portal hypertension: stellate cell contractility, endothelin, and nitric oxide. Hepatology. 25:2-5

46. Zhang, J.X., M. Bauer, and M.G. Clemens. 1995. Vessel and target cell specific actions of endothelin-1 and endothelin-3 in rat liver. Am. J. Physiol. 269:G269-G277.

47. Wake, K. 1980. Perisinusoidal stellate cells (fat-storing cells, interstitial cells, lipocytes) their related structure in and around the liver sinusoids, and vitamin A-storing cells in extrahepatic organs. Int. Rev. Cytol. 66:303-353.

48. McCuskey, R.S., and F.D. Reilly. 1993. Hepatic microvasculature: dynamic structure and its regulation. Semin. Liver Dis. 13:1-12.

49. Maass-Moreno, R., and C.F. Rothe. 1997. Distribution of pressure gradients along hepatic vasculature. Am. J. Physiol. 272:H2826-H2832.

50. Kaneda, K., W. Ekataksin, M. Sogawa, A. Matsumura, A. Cho, and N. Kawada. 1998. Endothelin-1-induced vasoconstriction causes a significant increase in portal pressure of rat liver: localized constrictive effect on the distal segment of preterminal portal venules as revealed by light and electron microscopy and serial reconstruction. Hepatology. 27:735-747.

51. Nakatani, T., L. Spolter, and K. Kobayashi. 1995. Arterial ketone body ratio as a parameter of hepatic mitochondrial redox state during and after hemorrhagic shock. World J. Surg. 19:592-596.

52. Pannen, B.H.J., F. Al-Adili, M. Bauer, M.G. Clemens, and K.K. Geiger. 1998. Role of endothelins and nitric oxide in hepatic reperfusion injury. Hepatology. 27:755-764.

53. Harbrecht, B.G., B. Wu, S.C. Watkins, H.P. Marshall, A.B. Peitzman, and T.R. Billiar. 1995. Inhibition of nitric oxide synthase during hemorrhagic shock increases hepatic injury. Shock. 4:332-337.

54. Kelly, E., N. Shah, N.N. Morgan, S.C. Watkins, A.B. Peitzman, and T.R. Billiar. 1994. Hemodynamic and molecular evidence that the vascular decompensation in hemorrhagic shock is not mediated by nitric oxide. Surg. Forum. 45:65-67. 